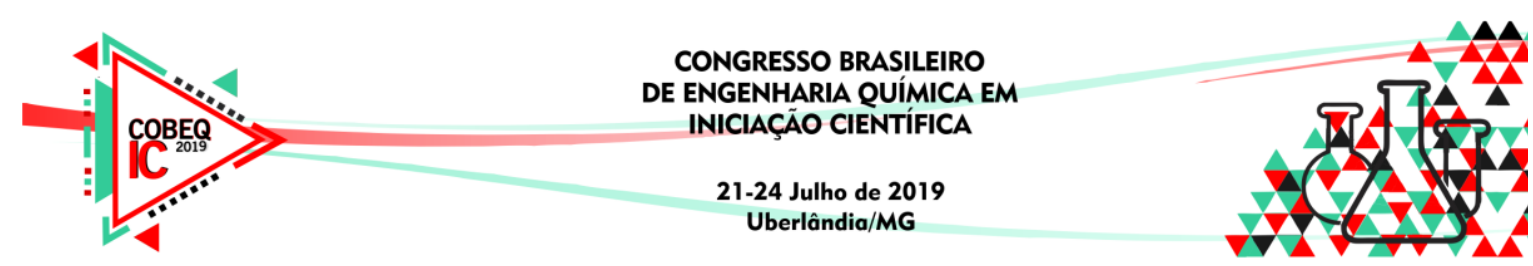

\title{
ESTUDOS QUÍMICOS E INCORPORAÇÃO DE EXTRATO GLICÓLICO DA FOLHA DE Campomanesia adamantium (o. Berg) EM CREME HIDRATANTE CICATRIZANTE
}

\author{
F. F. SILVA ${ }^{1}$, M. C. e $\operatorname{SILVA}^{1}$ e M. P. O. RAMOS ${ }^{2}$ \\ ${ }^{1}$ Centro Universitário de Patos de Minas, Faculdade de Engenharia Química \\ ${ }^{2}$ Professora Centro Universitário de Patos de Minas, Faculdade de Engenharia Química \\ E-mail para contato: franferreira02ff@gmail.com, mariaclarasilva.252@gmail.com, \\ perpetor@unipam.edu.br
}

\begin{abstract}
RESUMO - Campomanesia adamantium (Cambess) O. Berg, é popularmente conhecida como: gabiroba, guabiroba entre outros. A prospecção química ocorreu com testes específicos a partir da verificação de mudança de cor e ou formação de precipitados. Houve a determinação da atividade antioxidante pela captura do radical livre DPPH. O creme cicatrizante foi obtido utilizando formulação previamente proposta e os testes físico-químicos seguiram recomendações da Anvisa. Foi observada a presença de flavonóides, taninos, saponinas e quinonas nas folhas da $C$. adamantium. O extrato etanóico das folhas apresentou teor antioxidante em concentrações elevadas. A formulação do creme se mostrou estável quando submetida aos testes físico-químicos.
\end{abstract}

\section{INTRODUÇÃO}

A Campomanesia adamantium (Cambess) O. Berg, pertence à família Myrtaceae e é popularmente conhecida como: gabiroba, guabiroba, guabiroba-do-campo ou guavira. Encontrada comumente na região do cerrado brasileiro, com ocorrência de aparições no Paraguai e na Argentina, e maior concentração em Goiás (ARANTES e MONTEIRO, 2002). Estudos realizados nas folhas de espécies da mesma família que a $C$. adamantium indicaram a presença de flavonóides, taninos, saponinas e óleos essenciais, além da ação antioxidante, o que permite sua larga utilização na indústria fitoquímica.

Tem-se por objetivo deste trabalho a análise da presença de metabólitos especiais e atividade antioxidante no extrato etanóico da folha da $C$. adamantium, e a utilização do extrato glicólico em creme hidratante cicatrizante, e, análise físico-química do cosmético.

\section{MATERIAIS E MÉTODOS}

As amostras das folhas de $C$. adamantium foram obtidas da fazenda Santa Luiza, em uma área de cerrado, com localização Lat: -18,53'47’'S e Lng: -47,10’04”O, situada no município de Coromandel - MG, e posteriormente levadas ao laboratório Química Orgânica (bloco M, $2^{\circ}$ piso) do Centro Universitário de Patos de Minas - UNIPAM para secagem em estufa e outra parte levada ao Herbário do UNIPAM para a confecção da exsicata. As demais análises foram 


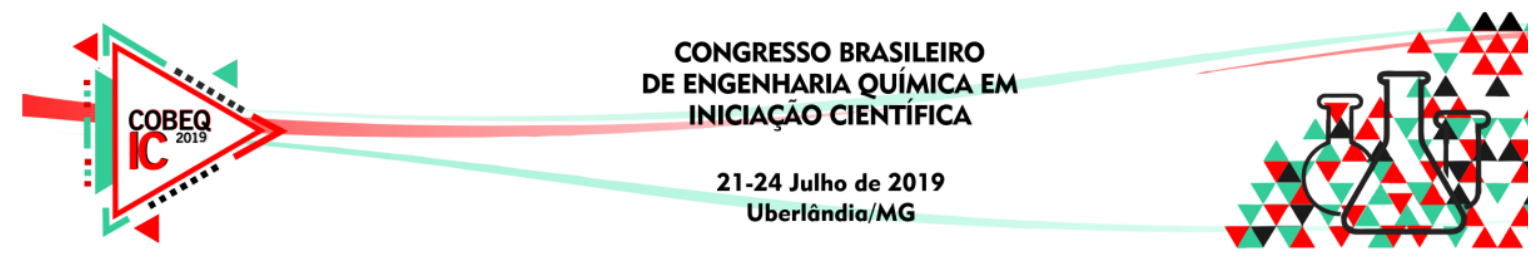

realizadas no laboratório de Química Orgânica e Central Analítica (bloco M, $2^{\circ}$ piso) e no laboratório de Engenharia Química (bloco I, $2^{\circ}$ piso).

\subsection{Metabólitos Especiais}

Todos os testes realizados para a identificação da presença de metabólitos especiais foram seguidos conforme a metodologia determinada pela Sociedade Brasileira de Farmacognosia (2009). Realizou-se testes de identificação para alcalóides, flavonóides, triterpenos e esteróides, cumarinas, glicosídeos cardiotônicos, quinonas e antraquinonas e taninos.

\subsection{Atividade Antioxidante}

Para determinação da atividade antioxidante pela captura do radical livre DPPH, seguiuse o procedimento determinado por Rosler et al. (2007), Souza et al. (2007), e Silva et al. (2009). A partir dos valores de absorbância foi utilizada a equação 1 para cálculo da porcentagem de atividade antioxidante $A A \%$.

$$
\% A A=\left(\frac{A_{0}-A}{A_{0}}\right) \times 100
$$

Onde $A A \%$ representa a porcentagem de atividade antioxidante, $A O$ é a absorbância do DPPH e $A$ é a absorbância da amostra com DPPH.

\subsection{Formulação do Creme Hidratante Cicatrizante}

O desenvolvimento da formulação envolveu a elaboração de uma emulsão, baseada na metodologia previamente descrita por Siqueira, (2017). A composição quali/quantitativa (\%) está descrita na tabela 1.

Tabela 1 - Formulação do Creme Hidratante Cicatrizante

\begin{tabular}{|l|l|l|l|}
\hline $\begin{array}{l}\text { Reagentes } \\
\text { Nome químico }\end{array}$ & Nome INCI & Nome comercial & (\%) \\
\hline Propilenoglicol & Propylene Glycol & - & 0,5 \\
\hline Phenonip & $\begin{array}{l}\text { Phenoxyethanol, Methylparaben, Ethylparaben, } \\
\text { Propylparaben, Butylparaben, Isobutylparaben }\end{array}$ & Fenoxietanol + Parabenos & 5,0 \\
\hline EDTA & Disodium EDTA & EDTA dissódico & 0,1 \\
\hline Base Croda & $\begin{array}{l}\text { Cetearyl Alcohol \&amp; Ceteareth 20; Mineral } \\
\text { Oil \&amp; Lanolin Alcohol; Petrolatum }\end{array}$ & Polawax Creme & 12,0 \\
\hline BHT & Butilidroxitolueno & - & 0,05 \\
\hline Fragrância & Fragrance & Essência de algodão & 0,5 \\
\hline Lanette N & Cetearyl Alcohol / Sodium Cetearyl Sulfate & Polybase N & 12,0 \\
\hline Estearato de Octila & Ethylhexyl Stearate & - & 7,14 \\
\hline Alantoina & Allantoin & - & 1,0 \\
\hline
\end{tabular}




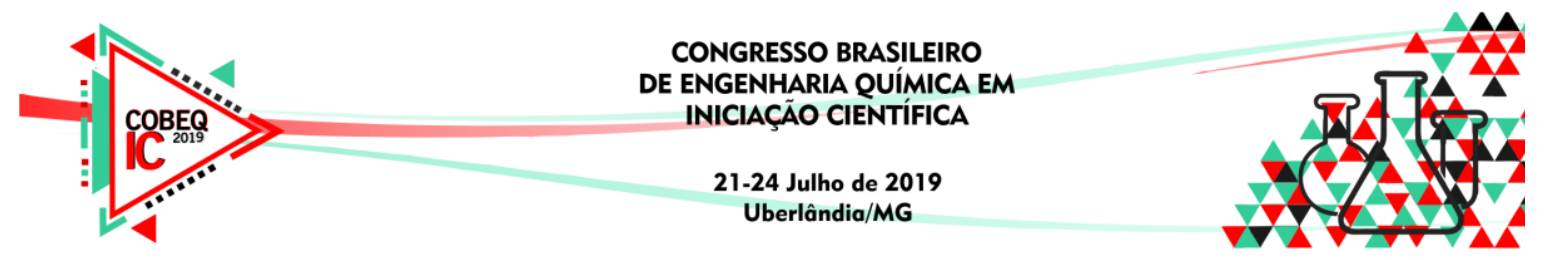

\begin{tabular}{|l|l|l|l|}
\hline $\begin{array}{l}\text { Reagentes } \\
\text { Nome químico }\end{array}$ & Nome INCI & Nome comercial & $\begin{array}{l}\text { Composição } \\
\text { (\%) }\end{array}$ \\
\hline D-Pantenol & Dexpanthenol & - & 2,0 \\
\hline Extrato glicólico & - & - & 7,0 \\
\hline Água deionizada & Aqua/Whater & - & 100 \\
\hline
\end{tabular}

\subsection{Testes Físico-Químicos do Creme Hidratante Cicatrizante}

Os testes físico-químicos foram realizados com base nos testes de estabilidade préestabelecidos pela Anvisa, no Guia de Estabilidade de Produtos Cosméticos (2004). Eles se subdividem em testes de estabilidade preliminar, sendo: testes de centrífuga, estufa $\left(40 \pm 2^{\circ} \mathrm{C}\right)$, geladeira $\left(5\right.$ a $\left.8^{\circ} \mathrm{C}\right), p \mathrm{H}$, densidade e viscosidade; e testes organolépticos, sendo estes: aspecto, cor e odor.

\section{RESULTADOS E DISCUSSÕES}

Os resultados obtidos na prospecção química de folhas de $C$. adamandtium estão apresentados na tabela 2, e foram comparados com os resultados de Markman et al., (2002) e Desoti et al., (2011), os quais analisaram as folhas da classe C. xanthocarpa (Myrtaceae) e Müller et al., (2012) que analisou as folhas da classe C. guazumaefolia (Camb.) Berg.

Tabela 2 - Resultados de metabólitos presentes no estudo e comparados com os obtidos na literatura

\begin{tabular}{|c|c|c|c|}
\hline Classe de Compostos & Reagentes & $\begin{array}{l}\text { Folha de C. } \\
\text { adamandtium } \\
(2018)\end{array}$ & $\begin{array}{l}\text { Resultados da } \\
\text { literatura }\end{array}$ \\
\hline \multirow{2}{*}{ Alcalóides } & Dragendorff & - & $-*$ \\
\hline & lodocloroplatinado & - & $-*$ \\
\hline \multirow{4}{*}{ Flavonóides } & Shinoda & +++ & $+* *$ \\
\hline & NP-PEG & +++ & NE \\
\hline & Cloreto de Alumínio & +++ & $+* *$ \\
\hline & Pew & +++ & NE \\
\hline Triterpenos e Esteróides & Liebermann-Burchard & - & $+++* *$ \\
\hline \multirow{2}{*}{ Cumarinas } & Solução de $\mathrm{NaOH}$ & - & $-* * *$ \\
\hline & Hidróxido de Potássio & - & $-* * *$ \\
\hline \multirow{3}{*}{ Glicosídeos Cardiotônicos } & Kedde & - & $-*$ \\
\hline & Liebermann-Burchard & - & + \\
\hline & Keller-Killiani & - & $-*$ \\
\hline \multirow{2}{*}{ Quinonas e Antraquinonas } & Borntranger direta & + & $-*$ \\
\hline & Borntranger com prévia hidrólise ácida & + & $-*$ \\
\hline Saponinas & Espuma persistente & + & $+^{*}$ \\
\hline
\end{tabular}




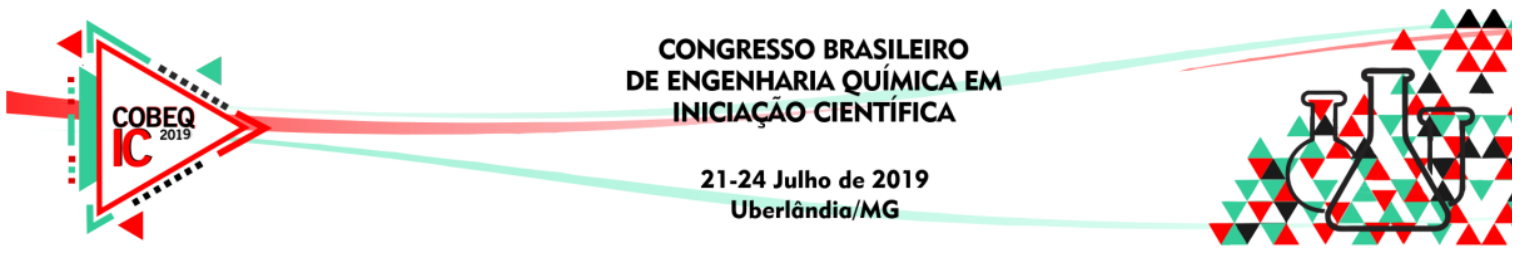

\begin{tabular}{|l|l|l|l|}
\hline \multirow{2}{*}{ Classe de Compostos } & Reagentes & $\begin{array}{l}\text { Folha de C. } \\
\text { adamandtium } \\
\mathbf{( 2 0 1 8 )}\end{array}$ & $\begin{array}{l}\text { Resultados da } \\
\text { literatura }\end{array}$ \\
\hline \multirow{3}{*}{ Taninos } & Gelatina & +++ & $+^{* *}$ \\
\cline { 2 - 4 } & $\mathrm{FeCl}_{3}$ & +++ & $+_{++}^{* *}$ \\
\cline { 2 - 4 } & Acetato de Cobre & +++ & $+_{++}^{* *}$ \\
\hline
\end{tabular}

Legenda: As cruzes indicam a intensidade da reação, sendo que: "+++" intensidade alta, "++" intensidade média, “+” intensidade baixa, “-” reação negativa. "*” testes comparados com Markman et al., (2002), “**” testes comparados com Desoti et al., (2011) e “***” testes comparados com Müller et al., (2012). Os testes NE indicam que foram não encontrados na literatura.

O $\mathrm{IC}_{50}$ encontrado para o extrato etanoico de $C$. adamantium foi de $116,424 \mu \mathrm{g} / \mathrm{mL}$, o valor encontrado na literatura para $\mathrm{IC}_{50}$ foi entre 40 e $160 \mu \mathrm{g} / \mathrm{mL}$ (RAMOS e CARDOSO, 2007), já o $\mathrm{IC}_{50}$ encontrado para a quercetina foi de $22,22 \mu \mathrm{g} / \mathrm{mL}$, o que era esperado, pois devido a sua grande atividade antioxidante, tende a precisar de um valor menor de concentração para decrescer a concentração inicial de DPPH em 50\%. A comparação feita entre a porcentagem de atividade antioxidante $A A \%$ do extrato da folha de $C$. adamantium e a quercetina é mostrada no Gráfico 1.

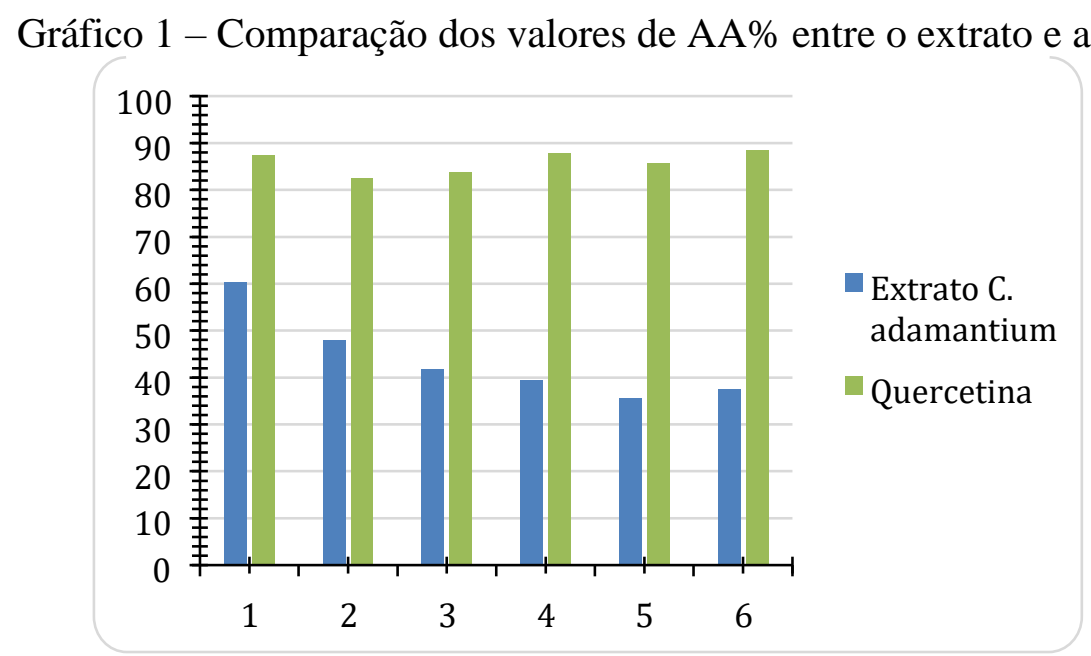

Os resultados dos testes de estabilidade estão descritos na tabela 3. A avaliação sob centrifugação, estufa e teste de geladeira e foram comparadas com os resultados obtidos por Bontorim (2009), a viscosidade com os resultados de Bontorim (2009) e Silva e Lopes (2017), o $p \mathrm{H}$ com os resultados de Bontorim (2009) e Pereira e Frasson (2007) e a densidade com os resultados de Silva e Lopes (2017). 


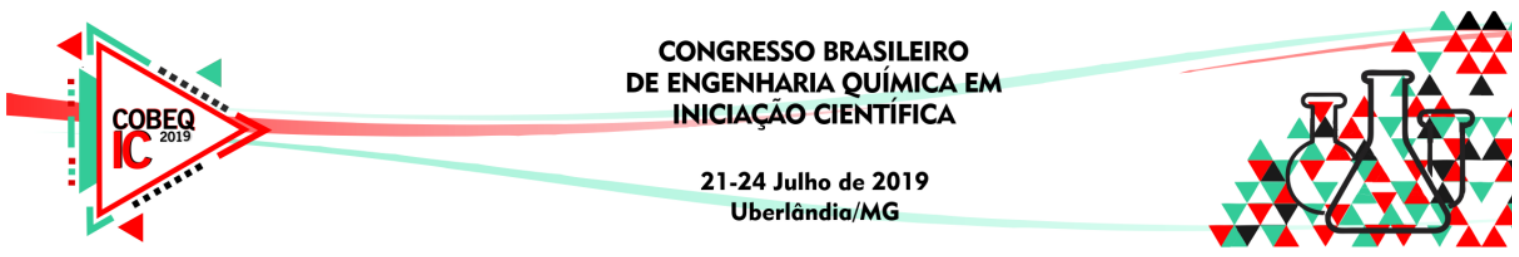

Tabela 3 - Resultados das análises de estabilidade

\begin{tabular}{|l|l|l|}
\hline Testes físico-químicos & $\begin{array}{l}\text { Creme hidratante } \\
\text { cicatrizante } \mathbf{( 2 0 1 8 )}\end{array}$ & Resultados da literatura \\
\hline Centrífuga & Sem alterações & $\begin{array}{l}\text { Creme sem separação de fases, massa brilhosa, homogênea, sem } \\
\text { grumos, livre de bolhas. }\end{array}$ \\
\hline Estufa $+40^{\circ} \mathrm{C}$ & Sem alterações & $\begin{array}{l}\text { Creme sem separação de fases, massa brilhosa, homogênea, sem } \\
\text { grumos, livre de bolhas. }\end{array}$ \\
\hline $\begin{array}{l}\text { Teste de Geladeira } 8 \text { a } \\
10^{\circ} \mathrm{C}\end{array}$ & Sem alterações & $\begin{array}{l}\text { Creme sem separação de fases, massa brilhosa, homogênea, sem } \\
\text { grumos, livre de bolhas. }\end{array}$ \\
\hline pH a $25^{\circ} \mathrm{C}$ & 5,97 & 5 a 6 \\
\hline Viscosidade & $7000 \mathrm{cP}$ & aprox. 5000 a $1000 \mathrm{cP}$ \\
\hline Densidade & $1,008 \mathrm{~g} / \mathrm{mL}$ & aprox. $0,800 \mathrm{~g} / \mathrm{mL}$ \\
\hline
\end{tabular}

A exsicata feita a partir da $C$. Adamantium obteve o número de tombo 218.14 .1 , e foi armazenada junto ao acervo de amostras de plantas secas do Laboratório de Ensino e Pesquisa Biológica, do UNIPAM.

\section{CONCLUSÃO}

A partir do estudo desenvolvido conclui-se que: com a adição do extrato glicólico da folha da $C$. Adamantium à formulação do creme hidratante cicatrizante se mostrou como importante e promissora forma farmacêutica com atividade farmacológica para o tratamento de processos de cicatrização, inflamações e feridas em geral, sendo possível verificar sua eficácia em posteriores testes.

\section{REFERÊNCIAS}

ARANTES, A. A.; MONTEIRO, R. A família Myrtaceae na Estação Ecológica do Panga, Uberlândia, Minas Gerais, Brasil. Instituto de Ciências Biológicas - UFMG, 2002.

BONTORIM, G.; Estudo de estabilidade de emulsão cosmética utilizando reologia e técnicas convencionais de análise. Universidade Federal do Paraná. Curitiba, 2009.

BRASIL, Ministério da Saúde, Agência Nacional de Vigilância Sanitária. Guia de Estabilidade de Produtos Cosméticos, maio de 2004.

DESOTI, V. C.; MALDANER, C. L.; CARLETTO, M. S.; HEINZ, A. A.; COELHO, M. S.; PIATI, D.; TIUMAN, T. S. Triagem fitoquímica e avaliação das atividades antimicrobiana e citotóxica de plantas medicinais nativas da região oeste do estado do Paraná. Arq. Ciênc. Saúde UNIPAR, Umuarama, jan./abr. 2011. 


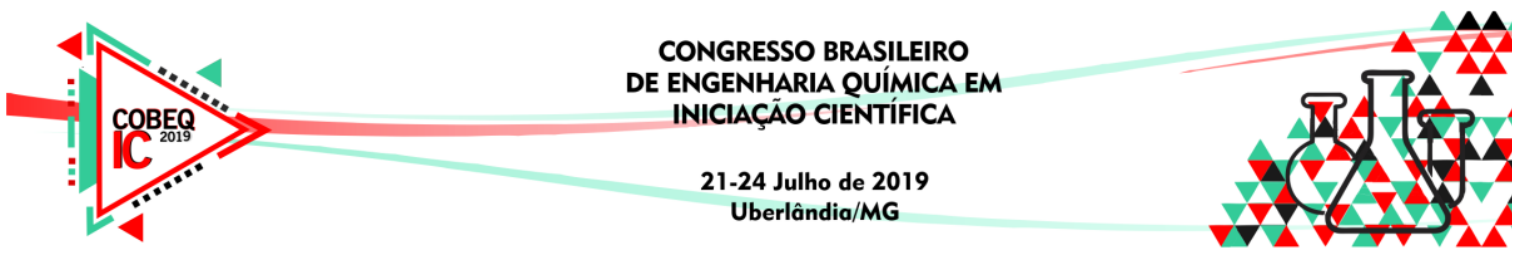

MARKMAN, B. E. O. Caracterização farmacognóstica de Campomanesia xanthocarpa Berg Myrtaceae. 2002.

MÜlleR, N. T. G.;FASOLO, D.; BERTÊ, R.; ELY,C. V.; HOLZ, D. T. Análise Fitoquímica das Folhas de Myrtaceae: Psidium Mattleianum Sabine e Campomanesia Guazumaefolia (Camb.) Berg. Vivências. Mai, 2012.

PEREIRA, D. C.; FRASSON, A. P. Z. Uso da aloe vera em produtos farmacêuticos e análise da estabilidade físico-química de creme aniônico contendo extrato glicólico desta planta. Revista Contexto \& Saúde. Universidade Regional do Noroeste do Estado do Rio Grande do Sul - Unijuí. 12, jan/jun. 2007.

RAMOS, D. D.; CARDOSO, C. A. Avaliação do potencial citotóxico e atividade antioxidante em Campomanesia adamantium (Cambess.) O. Berg (Myrtaceae). Revista Brasileira de Biociências. Universidade Federal do Rio Grande do Sul, 2007.

Revista Brasileira de Farmacognosia. (2009) Disponível em:<http://www.sbfgnosia.org.br/revista/> . Acesso em: 15, mai. 2018.

ROSLER, R.; Malta, L. G.; CARRASCO, L. C.; HOLANDA, R. B.; SILVA, A. C.; OLIVEIRA, M. C.; DEL RÉ, P. V.; JORGE, N. Utilização de extrato de cogumelo como antioxidante natural em óleo vegetal. Ciênc. agrotec., Lavras, jul./ago. 2009.

SILVA, T.; LOPES, L. L. B. T. Caracterização físico-química de uma formulação antiidade contendo ácido glicólico, produzida em farmácias de manipulação de Sete Lagoas- MG. Faculdade Ciências da Vida. Sete Lagoas - MG. 2017.

SIQUEIRA, A. P. N. D. F. Patos de Minas: Centro Universitário de Patos de Minas, 2017, 26 p. Apostila Fitocosmética.

SOUZA, C. A. S.; PASTORE, Glaucia Maria. Atividade antioxidante de frutas do cerrado. Ciência e Tecnologia de Alimentos. Campinas, SP. Jan/mar, 2007. 